\title{
Secure Regularized Zero Forcing for Multiuser MIMOME Channels
}

\author{
Saba Asaad*, Ali Bereyhi*, Ralf R. Müller*, and Rafael F. Schaefer ${ }^{\dagger}$ \\ ${ }^{*}$ Institute for Digital Communications, Friedrich-Alexander Universität Erlangen-Nürnberg, Germany \\ ${ }^{\dagger}$ Information Theory and Applications Chair, Technische Universität Berlin, Germany \\ \{saba.asaad, ali.bereyhi, ralf.r.mueller\}@fau.de, rafael.schaefer@tu-berlin.de
}

\begin{abstract}
This paper proposes a new linear precoding scheme for downlink transmission in MIMOME channels, referred to as secure regularized zero forcing. The scheme modifies regularized zero forcing precoding, such that the beamformers further suppress the information leakage towards the eavesdroppers. The proposed scheme is characterized in the large-system limit, and a closed-form expression for the achievable ergodic secrecy rate per user is derived. Numerical investigations demonstrate high robustness against the quality of eavesdroppers' channel.
\end{abstract}

Index Terms-MIMOME channels, linear precoding, physical layer security, large-system analysis.

\section{INTRODUCTION}

Conventional precoding schemes in multiple-input multipleoutput multiple-eavesdropper (MIMOME) channels [1] are often linear and independent of eavesdroppers' channel state information (CSI) [2]-[4]. The linearity constraint is mainly imposed for computational tractability. The independency from eavesdroppers' CSI further follows the fact that even simple beamforming towards the legitimate receivers suppresses the signal at the eavesdroppers effectively when the density of these malicious terminals in the network is low. In the asymptotic regime, this latter behavior is referred to as secrecy-forfree [5], [6] indicating that in massive multiple-input multipleoutput (MIMO) wiretap settings [7] with a fixed number of eavesdroppers, the information leakage vanishes as the number of antennas grows large, by using simple linear precoders.

Despite the above justifications, taking eavesdroppers' CSI into account at the precoder can result in significant performance enhancement, specially when 1) the density of malicious and legitimate terminals in the network is moderate or high, and 2) the quality of signals received by eavesdroppers is comparable to that of legitimate users. Such scenarios are likely to occur in current and next generations of mobile networks, due to the high number of mobile devices.

\section{A. Contributions}

In this paper, we propose a new linear precoding scheme for downlink transmission in MIMOME channels. The precoder

This work has been presented in the 2019 Asilomar Conference on Signals, Systems, and Computers. The link to the final version in the proceedings will be available later. follows the least-squares based approach, developed in [8][10], and extends the regularized zero forcing (RZF) precoding scheme [11] to MIMO systems with multiple eavesdroppers. To study the performance of the proposed scheme, we derive a closed-form expression for the achievable secrecy rate per user in the system when the number of transmit antennas, legitimate receivers and eavesdroppers grow large with fixed ratios. Our large-system analysis extends the earlier results in [2], [12] to the larger scope, and depicts tight consistency with simulations. Numerical investigations show that in contrast to RZF precoding, the proposed scheme is more robust against the quality of eavesdroppers' channel and report a significant performance gain when the quality of the channel to the eavesdroppers is better that that of legitimate users.

\section{B. Notations}

Throughout the paper, scalars, vectors, and matrices are represented by non-bold, bold lower case, and bold upper case letters, respectively. The real axis and complex plane are shown by $\mathbb{R}$ and $\mathbb{C}$, respectively. $\mathbf{H}^{\mathrm{H}}, \mathbf{H}^{*}$, and $\mathbf{H}^{\top}$ are the conjugate transpose, conjugate, and transpose of $\mathbf{H}$, respectively. $\|\mathbf{H}\|_{F}$ further denotes the Frobenius norm of $\mathbf{H} . \log (\cdot)$ is the binary logarithm, and $[x]^{+}:=\max \{0, x\}$. Expectation is denoted by $\mathbb{E}\{$.$\} , and \mathcal{C N}\left(\eta, \sigma^{2}\right)$ represents the complex Gaussian distribution with mean $\eta$ and variance $\sigma^{2}$. For brevity, the set $\{1, \ldots, N\}$ is abbreviated by $[N]$.

\section{Problem Formulation}

We consider a Gaussian multiuser MIMO wiretap channel with a base station (BS), $K$ legitimate receivers and $J$ eavesdroppers. The BS is equipped with a transmit array of size $M$, and the receiving terminals, i.e., the legitimate receivers and the eavesdroppers, are single-antenna. The system is assumed to perform in time-division duplexing (TDD) mode, and hence the uplink and downlink channels are reciprocal. The CSI is estimated in uplink training mode and is known at the BS, as well as the receiving terminals.

\section{A. System Model}

The BS intends to transmit messages $m_{k} \in\left[2^{N R_{k}}\right]$, for $k \in$ $[K]$, confidentially to legitimate receivers $k$. To this end, $m_{k}$ is first encoded into a codeword of length $N$, i.e. $\left[s_{k}(1), \ldots\right.$, $\left.s_{k}(N)\right]$, and then transmitted within $N$ transmission intervals 
over the channel as follows: At time instant $n$, the BS maps the encoded vector $\boldsymbol{s}(n)=\left[s_{1}(n), \ldots, s_{K}(n)\right]^{\top}$ to the transmit vector $\boldsymbol{x}(n) \in \mathbb{C}^{M}$ via the precoder $\operatorname{Prc}\{\cdot\}: \mathbb{C}^{K} \mapsto \mathbb{C}^{M}$, i.e. $\boldsymbol{x}(n)=\operatorname{Prc}\{\boldsymbol{s}(n)\}$, and transmits it via the antenna array.

We assume that the channel experiences quasi-static fading, such that its coherence time interval is larger than $N$ transmission intervals. Let $\mathbf{h}_{k} \in \mathbb{C}^{M}$ contain coefficients of the uplink channel between legitimate receiver $k$ and the BS. Due to the channel reciprocity, the receive signal in interval $n$ reads

$$
y_{k}(n)=\mathbf{h}_{k}^{\top} \boldsymbol{x}(n)+w_{k}(n)
$$

where $w_{k}(n)$ is complex white Gaussian noise with zero mean and variance $\sigma_{k}^{2}$, i.e., $w_{k}(n) \sim \mathcal{C N}\left(0, \sigma_{k}^{2}\right)$. After $N$ intervals, the receiver recovers $\hat{m}_{k}=\phi_{k}\left(\left\{y_{k}(n)\right\}\right)$, where $\left\{y_{k}(n)\right\}=$ $\left\{y_{k}(1), \ldots, y_{k}(N)\right\}$ and $\phi_{k}(\cdot)$ denotes the decoder.

For $j \in[J]$, the $j$-th eavesdropper observes

$$
z_{j}(n)=\mathbf{g}_{j}^{\top} \boldsymbol{x}(n)+v_{j}(n)
$$

by overhearing the channel. Here, $\mathbf{g}_{j} \in \mathbb{C}^{M}$ denotes the channel from the $j$-th eavesdropper to the $\mathrm{BS}$, and $v_{j}(n)$ is additive white Gaussian noise which reads $v_{j}(n) \sim \mathcal{C N}\left(0, \rho_{j}^{2}\right)$.

To guarantee secure transmission, we consider the worstcase scenario in which the eavesdroppers are cooperating. In this case, the secrecy rate tuple $\left(R_{1}, \ldots, R_{K}\right)$ is said to be achievable, if there exist a sequence of encoders and decoders, indexed by $N$, such that

$$
\begin{aligned}
& \lim _{N \uparrow \infty} \max _{k \in[K]}\left\{\operatorname{Pr}\left[\phi_{k}\left(\left\{y_{k}(n)\right\}\right) \neq m_{k}\right]\right\}=0 \\
& \lim _{N \uparrow \infty} \frac{1}{N} \mathrm{I}\left(\mathcal{S}\left(m_{1}, \ldots, m_{K}\right) ;\left\{z_{1}(n), \ldots, z_{J}(n)\right\}\right)=0
\end{aligned}
$$

for all $\mathcal{S}\left(m_{1}, \ldots, m_{K}\right) \subseteq\left\{m_{1}, \ldots, m_{K}\right\}$, where $\mathcal{S}(\cdot)$ is a subset of user messages. The constraint in $3 \mathrm{a}$ guarantees the reliability of transmissions towards the legitimate receivers. Moreover, (3b) indicates that the signals received by the eavesdroppers leak no information about any subset of the transmit messages.

\section{B. Achievable Secrecy Rate with Linear Precoding}

For linear precoders, the transmit vector is written as

$$
\boldsymbol{x}(n)=\mathbf{W} \boldsymbol{s}(n)
$$

for some precoding matrix $\mathbf{W}=\left[\mathbf{w}_{1}, \ldots, \mathbf{w}_{K}\right]$ satisfying the transmit power constraint $\mathbb{E}\left\{\operatorname{tr}\left\{\boldsymbol{x}^{\mathrm{H}} \boldsymbol{x}\right\}\right\} / M \leq P$. The vector $\mathbf{w}_{k} \in \mathbb{C}^{M}$, for $k \in[K]$, is referred to as the precoding vector of legitimate receiver $k$. Without loss of generality, we assume that $\mathbb{E}\left\{\boldsymbol{s}(n) \boldsymbol{s}^{\mathrm{H}}(n)\right\}=\mathbf{I}$.

Following the discussions in [2], [13], the secrecy rates

$$
R_{k}=\left[\log \left(\frac{1+\mathrm{SINR}_{k}}{1+\mathrm{ESNR}_{k}}\right)\right]^{+}
$$

are shown to be achievable for $k \in[K]$, where

$$
\begin{aligned}
\operatorname{SINR}_{k} & =\frac{\left|\mathbf{h}_{k}^{\top} \mathbf{w}_{k}\right|^{2}}{\sigma_{k}^{2}+\sum_{j=1, j \neq k}^{K}\left|\mathbf{h}_{k}^{\top} \mathbf{w}_{j}\right|^{2}}, \\
\mathrm{ESNR}_{k} & =\left\|\mathbf{K}_{\mathrm{Eve}} \mathbf{G w}_{k}\right\|^{2}
\end{aligned}
$$

$\mathbf{G}=\left[\mathbf{g}_{1}, \ldots, \mathbf{g}_{J}\right]^{\top}$ and $\mathbf{K}_{\mathrm{Eve}}=\operatorname{diag}\left\{1 / \rho_{1}, \ldots, 1 / \rho_{J}\right\}$. In the sequel, we consider the achievable rates in (5) as the metric to quantify the secrecy performance of the system.

The main goal of this study is to design an effective linear precoding scheme which takes into account the secrecy restrictions imposed by eavesdroppers. We address this objective by modifying RZF precoding [11], such that the information leakage to the eavesdroppers is efficiently suppressed at the precoding stage. For sake of brevity, we drop the time index, i.e. $n$, throughout the derivations in the remaining parts of this paper.

\section{SECURE RZF PRECODING}

In $\mathrm{RZF}$ precoding, the precoding matrix reads

$$
\mathbf{W}_{\mathrm{rzf}}(\zeta)=\sqrt{\frac{P}{\beta_{\mathrm{rzf}}(\zeta)}} \mathbf{A}(\zeta)
$$

for the shaping matrix

$$
\mathbf{A}(\zeta)=\mathbf{H}^{\mathrm{H}}\left(\mathbf{H} \mathbf{H}^{\mathrm{H}}+\zeta \mathbf{I}_{K}\right)^{-1}
$$

tuned by the regularizer $\zeta$ and the scaling factor

$$
\beta_{\mathrm{rzf}}(\zeta)=\frac{1}{M} \operatorname{tr}\left\{\mathbf{A}(\zeta) \mathbf{A}^{\mathrm{H}}(\zeta)\right\}
$$

which guarantees the satisfaction of the transmit power constraint. Here, $\mathbf{H}=\left[\mathbf{h}_{1}, \ldots, \mathbf{h}_{K}\right]^{\top}$ denotes the vector downlink channel. The shaping matrix in (8) performs regularized channel inversion. At $\zeta=0$, RZF precoding reduces to the zeroforcing scheme. In general, $\zeta$ is tuned such that a given performance metric, e.g. ergodic sum rate, is optimized.

\section{A. Alternative Formulation of RZF Precoding}

RZF precoding is alternatively observed as the regularized least-squares (RLS) solution to the following linear regression problem: Find matrix $\mathbf{W}$, such that the linear expansion $\mathbf{H} \boldsymbol{x}$ with $\boldsymbol{x}=\mathbf{W} s$ approximates an scaled version of $s$, i.e. $\psi \boldsymbol{s}$ for some $\psi$, with minimum least squared error (LSE), subject to $\mathbb{E}\left\{\|\boldsymbol{x}\|^{2}\right\} \leq P$. Following the method of RLS, the solution to this problem is given by minimizing

$$
\operatorname{RSS}(\mathbf{W}):=\mathbb{E}_{\boldsymbol{s}}\left\{\|\mathbf{H W} \boldsymbol{s}-\psi \boldsymbol{s}\|^{2}\right\},
$$

known as the residual sum of squares (RSS), penalized by the power constraint. In other words, $\mathbf{W}$ is found by

$$
\begin{aligned}
\mathbf{W} & =\underset{\mathbf{X} \in \mathbb{C}^{M \times K}}{\operatorname{argmin}} \operatorname{RSS}(\mathbf{X})+\zeta \mathbb{E}_{s}\left\{\|\mathbf{X} s\|^{2}\right\} \\
& =\underset{\mathbf{X} \in \mathbb{C}^{M \times K}}{\operatorname{argmin}} \operatorname{tr}\left\{\mathbf{X}^{\mathrm{H}}\left(\mathbf{H}^{\mathrm{H}} \mathbf{H}+\zeta \mathbf{I}_{M}\right) \mathbf{X}-2 \psi \Re\{\mathbf{H X}\}\right\} \\
& =\psi \mathbf{A}(\zeta)
\end{aligned}
$$

where $\zeta$ is a Lagrange multiplier. By considering the transmit power constraint, 11b reduces to (7). Extension of this RLS based approach to other constraints leads to generalized least squared error (GLSE) precoding which has been proposed and studied in [8]-[10]. 


\section{B. RLS-Based Precoding with Security Constraints}

Following the RLS interpretation of RZF precoding, the secrecy constraint can be further imposed at the transmit side by penalizing the RSS term. To illustrate this point, let

$$
f_{\text {Eve }}(\mathbf{W}): \mathbb{C}^{M \times K} \mapsto \mathbb{R}_{0}^{+}
$$

quantify the information leakage when the linear precoder $\mathbf{W}$ is employed. Let $f_{\text {Eve }}(\cdot)$ be proportional to the information leakage meaning that $f_{\text {Eve }}\left(\mathbf{W}_{1}\right) \leq f_{\text {Eve }}\left(\mathbf{W}_{2}\right)$ indicates that $\boldsymbol{s}$ is estimated from the overheard signals in $z_{1}=\mathbf{G W}_{1} \boldsymbol{s}+\boldsymbol{v}$ with higher error probability compared to $z_{2}=\mathbf{G W}_{2} \boldsymbol{s}+\boldsymbol{v}$.

Given $f_{\text {Eve }}(\cdot)$, a secrecy constraint can be imposed on the system by restricting the precoding matrix $\mathbf{W}$ to satisfy

$$
f_{\text {Eve }}(\mathbf{W}) \leq L
$$

for some information leakage $L$. The RLS formulation in this case can be modified by penalizing the RSS term with both the power and secrecy constraints. That means $\mathbf{W}$ is set to

$$
\mathbf{W}=\underset{\mathbf{X} \in \mathbb{C}^{M \times K}}{\operatorname{argmin}} \operatorname{RSS}(\mathbf{X})+\lambda \mathbb{E}_{s}\left\{\|\mathbf{X} \boldsymbol{s}\|^{2}\right\}+\theta f_{\text {Eve }}(\mathbf{X})
$$

for some tunable factors $\lambda$ and $\theta$. The optimization in (14) simultaneously reduces the LSE at the legitimate terminals and the leakage towards the eavesdroppers.

Deriving a function which analytically characterizes the information leakage is not a tractable task. Nevertheless, one can consider an alternative metric which is proportional to the capability of the eavesdroppers in decoding the information. To find such a metric, we note that in the ideal case with significantly narrow beamforming, we desire to have

$$
\left|\mathbf{g}_{j}^{\top} \mathbf{w}_{k}\right|=0,
$$

for $j \in[J]$ and $k \in[K]$. This indicates that a natural choice for $f_{\mathrm{Eve}}(\cdot)$ is

$$
\begin{aligned}
f_{\text {Eve }}(\mathbf{W}) & =\sum_{j=1}^{J} \sum_{k=1}^{K}\left|\mathbf{g}_{j}^{\top} \mathbf{w}_{k}\right|^{2} \\
& =\|\mathbf{G} \mathbf{W}\|_{F}^{2}=\operatorname{tr}\left\{\mathbf{W}^{\mathrm{H}} \mathbf{G}^{\mathrm{H}} \mathbf{G W}\right\}
\end{aligned}
$$

By substituting (16b), (14) reduces to a convex optimization problem whose solution is $\mathbf{W}=\psi \mathbf{A}(\lambda, \theta)$ where

$$
\mathbf{A}(\lambda, \theta)=\left(\mathbf{H}^{\mathrm{H}} \mathbf{H}+\theta \mathbf{G}^{\mathrm{H}} \mathbf{G}+\lambda \mathbf{I}_{M}\right)^{-1} \mathbf{H}^{\mathrm{H}} .
$$

By restricting the transmit power to $P$, the secure RZF (SRZF) precoder is concluded as

$$
\mathbf{W}_{\text {srzf }}(\lambda, \theta)=\sqrt{\frac{P}{\beta_{\text {srzf }}(\lambda, \theta)}} \mathbf{A}(\lambda, \theta)
$$

with

$$
\beta_{\text {srzf }}(\lambda, \theta)=\frac{1}{M} \operatorname{tr}\left\{\mathbf{A}(\lambda, \theta) \mathbf{A}^{\mathrm{H}}(\lambda, \theta)\right\} .
$$

Note that $\mathbf{W}_{\text {srzf }}(\zeta, 0)=\mathbf{W}_{\mathrm{rzf}}(\zeta)$. In fact, the SRZF scheme utilizes the CSI of the malicious terminals and modifies RZF beamformers, such that leakage to the eavesdroppers is further suppressed. In general, $\theta$ and $\lambda$ are tuned such that a given performance metric, e.g. ergodic sum rate, is optimized.

\section{LARGE-SYSTEM ANALYSIS}

In this section, the large-system performance of the proposed precoding scheme is characterized. To this end, we consider a scenario in which the number of transmit antennas $M$, number of legitimate receivers $K$ and number of eavesdroppers $J$ are significantly large; however, the ratios

$$
\begin{aligned}
\alpha_{1} & =\frac{K}{M} \\
\alpha_{\mathrm{o}} & =\frac{J}{M}
\end{aligned}
$$

are constant. We refer to $\alpha_{1}$ as the legitimate channel load, and to $\alpha_{\mathrm{o}}$ as the overhearing channel load. For sake of brevity, we further assume that

- For $k \in[K]$ and $j \in[J], \mathbf{h}_{k}$ and $\mathbf{g}_{j}$ are are independent and identically distributed (i.i.d.) Gaussian vectors with zero mean and variance $1 / M$.

- $\rho_{j}^{2}=\rho^{2}$ for $j \in[J]$, and $\sigma_{k}^{2}=\sigma^{2}$ for $k \in[K]$.

To start the derivations, let us define

$$
\mathbf{Q}=\mathbf{H}^{\mathrm{H}} \mathbf{H}+\theta \mathbf{G}^{\mathrm{H}} \mathbf{G}+\lambda \mathbf{I}_{M} .
$$

Hence, the $k$-th beamformer of the SRZF precoder reads

$$
\mathbf{w}_{k}=\sqrt{\frac{P}{\beta_{\text {srff }}(\lambda, \theta)}} \mathbf{Q}^{-1} \mathbf{h}_{k}^{*} .
$$

As a result, the signal to interference plus noise ratio (SINR) at legitimate receiver $k$ reads

$$
\mathrm{SINR}_{k}=\frac{\mu_{1} U_{k}}{\beta_{\text {srzf }}(\lambda, \theta)+\mu_{1} I_{k}} .
$$

where $\mu_{1}:=P / \sigma^{2}$ is the receive signal to noise ratio (SNR) at the legitimate terminals, and

$$
\begin{aligned}
U_{k} & =\left|\mathbf{h}_{k}^{\top} \mathbf{Q}^{-1} \mathbf{h}_{k}^{*}\right|^{2}, \\
I_{k} & =\sum_{j=1, j \neq k}^{K}\left|\mathbf{h}_{k}^{\top} \mathbf{Q}^{-1} \mathbf{h}_{j}^{*}\right|^{2} .
\end{aligned}
$$

Using the Sherman-Morrison lemma, it is shown that

$$
\begin{aligned}
U_{k} & =\left(\frac{\mathbf{h}_{k}^{\top} \mathbf{Q}_{k}^{-1} \mathbf{h}_{k}^{*}}{1+\mathbf{h}_{k}^{\top} \mathbf{Q}_{k}^{-1} \mathbf{h}_{k}^{*}}\right)^{2} \\
I_{k} & =\sum_{j=1, j \neq k}^{K} \frac{\left|\mathbf{h}_{k}^{\top} \mathbf{Q}_{k, j}^{-1} \mathbf{h}_{j}^{*}\right|^{2}}{\left(1+\mathbf{h}_{k}^{\top} \mathbf{Q}_{k}^{-1} \mathbf{h}_{k}^{*}\right)^{2}\left(1+\mathbf{h}_{j}^{\top} \mathbf{Q}_{k, j}^{-1} \mathbf{h}_{j}^{*}\right)^{2}}
\end{aligned}
$$

where $\mathbf{Q}_{k}:=\mathbf{Q}-\mathbf{h}_{k}^{*} \mathbf{h}_{k}^{\top}$ and $\mathbf{Q}_{k, j}:=\mathbf{Q}-\mathbf{h}_{k}^{*} \mathbf{h}_{k}^{\top}-\mathbf{h}_{j}^{*} \mathbf{h}_{j}^{\top}$. For scaling factor $\beta_{\text {srzf }}(\lambda, \theta)$, we further can write

$$
\begin{aligned}
\beta_{\text {srzf }}(\lambda, \theta) & =\frac{1}{M} \operatorname{tr}\left\{\mathbf{A}(\lambda, \theta) \mathbf{A}^{\mathrm{H}}(\lambda, \theta)\right\} \\
& =\frac{1}{M} \operatorname{tr}\left\{\mathbf{H} \mathbf{Q}^{-2} \mathbf{H}^{\mathrm{H}}\right\} \\
& =\frac{1}{M} \sum_{k=1}^{K} \mathbf{h}_{k}^{\top} \mathbf{Q}^{-2} \mathbf{h}_{k}^{*} \\
& \stackrel{\star}{=} \frac{1}{M} \sum_{k=1}^{K} \frac{\mathbf{h}_{k}^{\top} \mathbf{Q}_{k}^{-2} \mathbf{h}_{k}^{*}}{\left(1+\mathbf{h}_{k}^{\top} \mathbf{Q}_{k}^{-1} \mathbf{h}_{k}^{*}\right)^{2}}
\end{aligned}
$$


where $\star$ follows from the Sherman-Morrison lemma.

For $\mathrm{ESNR}_{k}$, we can similarly write

$$
\operatorname{ESNR}_{k}=\frac{1}{\rho^{2}}\left\|\mathbf{G w}_{k}\right\|^{2}=\frac{\mu_{\mathrm{o}} L_{k}}{\beta_{\text {srzf }}(\lambda, \theta)}
$$

where $\mu_{\mathrm{o}}:=P / \rho^{2}$ is the receive SNR at the eavesdroppers, and

$$
\begin{aligned}
L_{k} & =\mathbf{h}_{k}^{\top} \mathbf{Q}^{-1} \mathbf{G}^{\mathrm{H}} \mathbf{G} \mathbf{Q}^{-1} \mathbf{h}_{k}^{*} \\
& =\frac{\mathbf{h}_{k}^{\top} \mathbf{Q}_{k}^{-1} \mathbf{G}^{\mathrm{H}} \mathbf{G} \mathbf{Q}_{k}^{-1} \mathbf{h}_{k}^{*}}{\left(1+\mathbf{h}_{k}^{\top} \mathbf{Q}_{k}^{-1} \mathbf{h}_{k}^{*}\right)^{2}} \\
& =\frac{1}{\left(1+\mathbf{h}_{k}^{\top} \mathbf{Q}_{k}^{-1} \mathbf{h}_{k}^{*}\right)^{2}} \operatorname{tr}\left\{\mathbf{G} \mathbf{Q}_{k}^{-1} \mathbf{h}_{k}^{*} \mathbf{h}_{k}^{\top} \mathbf{Q}_{k}^{-1} \mathbf{G}^{\mathrm{H}}\right\} \\
& =\frac{1}{\left(1+\mathbf{h}_{k}^{\top} \mathbf{Q}_{k}^{-1} \mathbf{h}_{k}^{*}\right)^{2}} \sum_{j=1}^{J}\left|\mathbf{g}_{j} \mathbf{Q}_{k}^{-1} \mathbf{h}_{k}^{*}\right|^{2} \\
& =\frac{1}{\left(1+\mathbf{h}_{k}^{\top} \mathbf{Q}_{k}^{-1} \mathbf{h}_{k}^{*}\right)^{2}} \sum_{j=1}^{J} \frac{\left|\mathbf{g}_{j} \boldsymbol{\Gamma}_{k, j}^{-1} \mathbf{h}_{k}^{*}\right|^{2}}{\left(1+\theta \mathbf{g}_{j} \boldsymbol{\Gamma}_{k, j}^{-1} \mathbf{g}_{j}^{*}\right)^{2}}
\end{aligned}
$$

Here, we define $\boldsymbol{\Gamma}_{k, j}:=\mathbf{Q}-\mathbf{h}_{k}^{*} \mathbf{h}_{k}^{\top}-\theta \mathbf{g}_{j}^{*} \mathbf{g}_{j}^{\top}$.

\section{A. Asymptotics via Free Probability}

To determine the asymptotic limits, we note that

1) Any two independent Hermitian random matrices, which are unitarily invariant, are asymptotically free [14]. This result indicates that

- $\mathbf{h}_{k}^{*} \mathbf{h}_{k}^{\top}$ and $\mathbf{Q}_{k}^{-1}$ are asymptotically free for $k \in[K]$.

- $\mathbf{h}_{k}^{*} \mathbf{h}_{k}^{\top}$ and $\mathbf{Q}_{k, j}^{-1}$ are asymptotically free for $k, j \in[K]$.

- $\mathbf{h}_{k}^{*} \mathbf{h}_{k}^{\top}$ and $\boldsymbol{\Gamma}_{k, j}^{-1}$ are asymptotically free for $k \in[K]$.

- $\mathbf{g}_{j}^{*} \mathbf{g}_{j}^{\top}$ and $\boldsymbol{\Gamma}_{k, j}^{-1}$ are asymptotically free for $j \in[J]$.

2) $\mathbf{Q}_{k}, \mathbf{Q}_{k, j}$, and $\boldsymbol{\Gamma}_{k, j}$ are single-rank perturbations of $\mathbf{Q}$. As a result, the asymptotic distribution of their eigenvalues is similar to that of $\mathbf{Q}$.

3) Defining the matrix $\mathbf{T}:=\mathbf{H}^{\mathrm{H}} \mathbf{H}+\theta \mathbf{G}^{\mathrm{H}} \mathbf{G}$, we have

$$
\mathbf{Q}=\mathbf{T}+\lambda \mathbf{I}_{M} .
$$

Let us denote the asymptotic distribution of the eigenvalues of $\mathbf{T}$ with $\mathrm{p}_{\mathbf{T}}(t)$. The eigenvalues of $\mathbf{Q}$ are shifted versions of the eigenvalues of $\mathbf{T}$ and are asymptotically distributed by $\mathrm{p}_{\mathbf{T}}(t-\lambda)$.

Considering the above findings, we can write

$$
\begin{aligned}
& \lim _{M \uparrow \infty} \frac{\mathbf{h}_{k}^{\top} \mathbf{Q}_{k}^{-1} \mathbf{h}_{k}^{*}}{M}=\lim _{M \uparrow \infty} \frac{\operatorname{tr}\left\{\mathbf{Q}_{k}^{-1} \mathbf{h}_{k}^{*} \mathbf{h}_{k}^{\top}\right\}}{M} \\
& \stackrel{\dagger}{=} \lim _{M \uparrow \infty} \frac{\operatorname{tr}\left\{\mathbf{Q}_{k}^{-1}\right\}}{M} \times \lim _{M \uparrow \infty} \frac{\operatorname{tr}\left\{\mathbf{h}_{k}^{*} \mathbf{h}_{k}^{\top}\right\}}{M}
\end{aligned}
$$

where $\dagger$ follows the fact that $\mathbf{h}_{k}^{*} \mathbf{h}_{k}^{\top}$ and $\mathbf{Q}_{k}^{-1}$ are asymptotically free. Noting that

$$
\lim _{M \uparrow \infty} \operatorname{tr}\left\{\mathbf{h}_{k}^{*} \mathbf{h}_{k}^{\top}\right\}=\lim _{M \uparrow \infty}\left\|\mathbf{h}_{k}\right\|^{2}=1,
$$

we have

$$
\begin{aligned}
\lim _{M \uparrow \infty} \mathbf{h}_{k}^{\top} \mathbf{Q}_{k}^{-1} \mathbf{h}_{k}^{*} & =\lim _{M \uparrow \infty} \frac{1}{M} \operatorname{tr}\left\{\mathbf{Q}_{k}^{-1}\right\} \\
& =\mathbb{E}_{T}\left\{\frac{1}{T+\lambda}\right\}=G_{\mathbf{T}}(-\lambda)
\end{aligned}
$$

for some $T \sim \mathrm{p}_{\mathbf{T}}$ with Stieltjes transform $G_{\mathbf{T}}(\cdot)$ defined as

$$
G_{\mathbf{T}}(s)=\int \frac{\mathrm{p}_{\mathbf{T}}(t) \mathrm{d} t}{t-s} .
$$

Clearly, the limit does not depend on $k$. As a result,

$$
U_{k}=\left(\frac{G_{\mathbf{T}}(-\lambda)}{1+G_{\mathbf{T}}(-\lambda)}\right)^{2}
$$

for $k \in[K]$. By same lines of derivations, we have

$$
\begin{aligned}
I_{k} & =\alpha_{1}\left(1+\mathbb{E}_{T}\left\{\frac{1}{T+\lambda}\right\}\right)^{-4} \mathbb{E}_{T}\left\{\frac{1}{(T+\lambda)^{2}}\right\} \\
& =\frac{\alpha_{1} G_{\mathbf{T}}^{\prime}(-\lambda)}{\left(1+G_{\mathbf{T}}(-\lambda)\right)^{4}},
\end{aligned}
$$

and

$$
\begin{aligned}
\beta_{\text {srzf }}(\lambda, \theta) & =\frac{\alpha_{1} G_{\mathbf{T}}^{\prime}(-\lambda)}{\left(1+G_{\mathbf{T}}(-\lambda)\right)^{2}} \\
L_{k} & =\frac{\alpha_{\mathrm{o}} G_{\mathbf{T}}^{\prime}(-\lambda)}{\left(1+G_{\mathbf{T}}(-\lambda)\right)^{2}\left(1+\theta G_{\mathbf{T}}(-\lambda)\right)^{2}} .
\end{aligned}
$$

Consequently, $\mathrm{SINR}_{k}$ in the large-system limit converges to

$$
\operatorname{SINR}_{\text {asy }}=\frac{\mu_{\mathrm{l}} G_{\mathbf{T}}^{2}(-\lambda)\left(1+G_{\mathbf{T}}(-\lambda)\right)^{2}}{\alpha_{1} G_{\mathbf{T}}^{\prime}(-\lambda)\left[\mu_{1}+\left(1+G_{\mathbf{T}}(-\lambda)\right)^{2}\right]}
$$

and the asymptotic limit of $\mathrm{ESNR}_{k}$ is

$$
\mathrm{ESNR}_{\text {asy }}=\frac{\mu_{\mathrm{o}} \alpha_{\mathrm{o}}}{\alpha_{1}\left(1+\theta G_{\mathbf{T}}(-\lambda)\right)^{2}} .
$$

To determine this limit for particular scenarios, we further need to determine the Stieltjes transform $G_{\mathbf{T}}(\cdot)$.

Remark 1: Note that throughout the derivations, we did not utilize the Gaussianity of the channel matrices. In fact, the results in (38) and (39) are valid for any unitarily invariant $\mathbf{H}$ and $\mathbf{G}$ whose row vectors are jointly independent.

\section{B. Stieltjes Transform of $\mathbf{T}$}

To find the Stieltjes Transform of $\mathbf{T}$, we rewrite $\mathbf{T}$ as

$$
\mathbf{T}=\tilde{\mathbf{H}}^{\mathrm{H}} \mathbf{D} \tilde{\mathbf{H}}
$$

where $\tilde{\mathbf{H}}$ is defined as

$$
\tilde{\mathbf{H}}:=\left[\mathbf{h}_{1}, \ldots, \mathbf{h}_{K}, \mathbf{g}_{1}, \ldots, \mathbf{g}_{J}\right]^{\top},
$$

and $\mathbf{D} \in \mathbb{R}^{(K+J) \times(K+J)}$ is a diagonal matrix whose first $K$ diagonal entries are one and the rest are $\theta$. Noting that $\tilde{\mathbf{H}}$ fulfills the conditions for the deformed quarter circle law, one can invoke the Silverstein-Bai result [15], [16] and write

$$
G_{\mathbf{T}}(s)=\frac{1}{-s+\left(\alpha_{1}+\alpha_{\mathrm{o}}\right) \int \frac{y \mathrm{p}_{\mathbf{D}}(y) \mathrm{d} y}{1+y G_{\mathbf{T}}(s)}}
$$




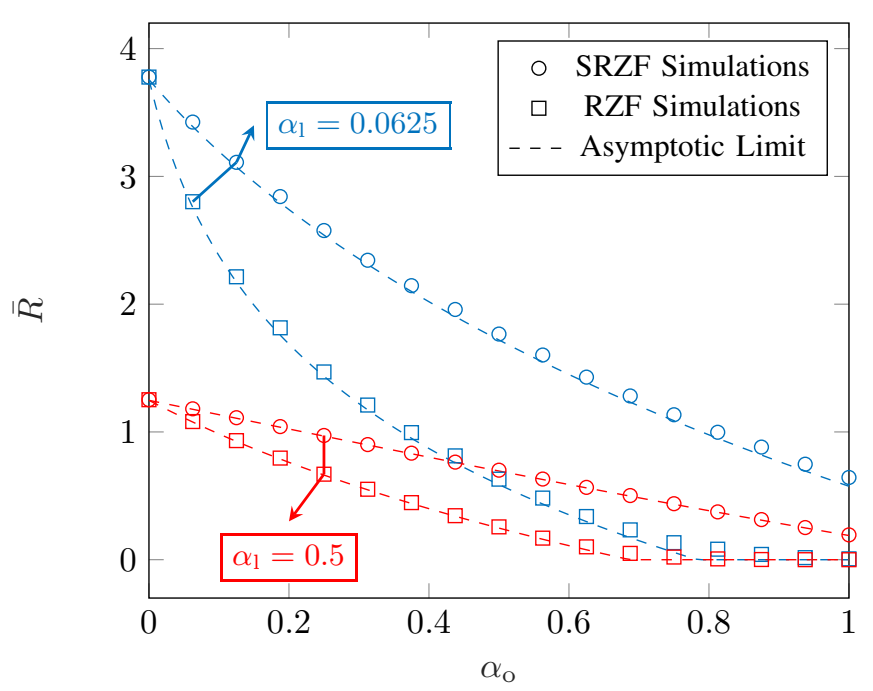

Fig. 1: Average ergodic secrecy rate vs. the overhearing channel load. The simulations are given for $M=128$ and $\log \mu_{1}=\log \mu_{\mathrm{o}}=0$ dB. For SRZF precoding, $\lambda=\theta=1$, and $\zeta=1$ in RZF.

where $\mathrm{p}_{\mathbf{D}}(\cdot)$ denotes the distributions of the diagonal entries of $\mathbf{D}$ and reads

$$
\mathrm{p}_{\mathbf{D}}(y)=\frac{\alpha_{1}}{\alpha_{1}+\alpha_{\mathrm{o}}} \delta(y-1)+\frac{\alpha_{\mathrm{o}}}{\alpha_{1}+\alpha_{\mathrm{o}}} \delta(y-\theta) .
$$

Substituting into (42), we finally conclude

$$
1+s G_{\mathbf{T}}(s)=\alpha_{1} \frac{G_{\mathbf{T}}(s)}{1+G_{\mathbf{T}}(s)}+\alpha_{\mathrm{o}} \frac{\theta G_{\mathbf{T}}(s)}{1+\theta G_{\mathbf{T}}(s)}
$$

By taking derivative from the both sides of this equation, we further conclude

$$
G_{\mathbf{T}}^{\prime}(s)=\frac{G_{\mathbf{T}}(s)}{-s+\frac{\alpha_{1}}{\left(1+G_{\mathbf{T}}(s)\right)^{2}}+\frac{\theta \alpha_{\mathrm{o}}}{\left(1+\theta G_{\mathbf{T}}(s)\right)^{2}}} .
$$

As a result, $G_{\mathbf{T}}(-\lambda)$ is found as the positive solution of

$$
\lambda+\frac{\alpha_{1}}{1+\mathrm{x}}+\frac{\theta \alpha_{\mathrm{o}}}{1+\theta \mathrm{x}}=\frac{1}{\mathrm{x}}
$$

and $G_{\mathbf{T}}^{\prime}(-\lambda)$ is calculated by

$$
G_{\mathbf{T}}^{\prime}(-\lambda)=\frac{\mathrm{x}}{\lambda+\frac{\alpha_{\mathrm{l}}}{(1+\mathrm{x})^{2}}+\frac{\theta \alpha_{\mathrm{o}}}{(1+\theta \mathrm{x})^{2}}} .
$$

By standard lines of derivations, one can show that the results recover the earlier derivations in [2], [12].

\section{NUMERICAL INVESTIGATIONS}

To investigate the performance of the proposed precoding scheme and validate the analytic derivations, we study sample scenarios via numerical simulations. As an overall measure of performance, we define the average ergodic secrecy rate as

$$
\bar{R}=\frac{1}{K} \sum_{k=1}^{K} \mathbb{E}\left\{R_{k}\right\}
$$

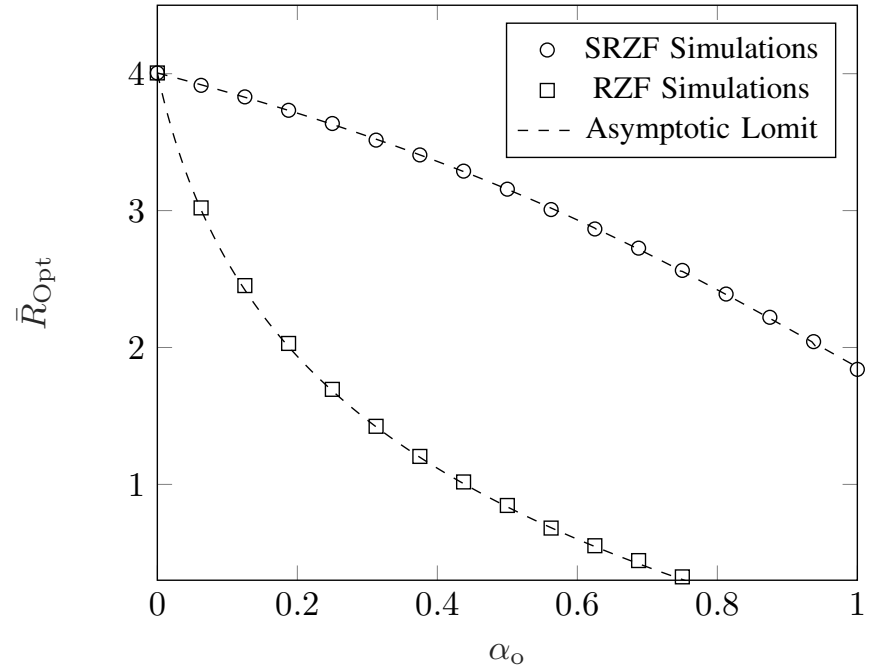

Fig. 2: Optimized average ergodic secrecy rate vs. the overhearing channel load. The simulations are given for $M=128$. The results are sketched for $\log \mu_{1}=\log \mu_{\mathrm{o}}=0 \mathrm{~dB}$ and $\alpha_{1}=0.0625$.

Following the asymptotic analysis, as $M$ grows large, $R_{k} \rightarrow$ $\mathbb{E}\left\{R_{k}\right\}$ and $\mathbb{E}\left\{R_{k}\right\} \rightarrow \bar{R}$ for $k \in[K]$. Thus,

$$
\lim _{M \uparrow \infty} \bar{R}=\left[\log \left(\frac{1+\text { SINR }_{\text {asy }}}{1+\text { ESNR }_{\text {asy }}}\right)\right]^{+} .
$$

To validate the large-system results, we sketch in Fig. 1 1 the average ergodic secrecy rate against the overhearing channel load $\alpha_{\mathrm{o}}$ for two scenarios; namely, a scenario with high user density, i.e. $\alpha_{1}=0.5$, and a scenario with low user density, i.e. $\alpha_{1}=0.0625$. In both scenarios, the SNR at all receive terminals is set to one, i.e. $\log \mu_{1}=\log \mu_{\mathrm{o}}=0 \mathrm{~dB}$. For sake of comparison, the results are given for both the SRZF and RZF schemes, where in the SRZF precoder $\lambda=\theta=1$, and in RZF $\zeta=1$. The entries of $\mathbf{H}$ and $\mathbf{G}$ are generated i.i.d. with $\mathcal{C N}(0,1 / M)$. The figure shows the simulations for $M=128$ transmit antennas, as well as the results given via asymptotic analyses. It is seen that the analytic derivations are tightly consistent with the simulation results. From the figure, it is further observed that for the given setting the proposed scheme is constantly outperforming RZF. This observation however needs further investigations, as in this setting the tunable parameters, i.e. $\lambda$ and $\theta$, are kept fixed.

\section{A. Optimal Tuning of SRZF Precoding}

Following the tight consistency of analytical results, seen in Fig. 11 we tune the SRZF precoder by optimizing the asymptotic limit of the average ergodic secrecy rate. In other words, for a given setting, we find $\lambda$ and $\theta$ such that the limiting value of $\bar{R}$, given in (49), is maximized. We denote this maximum value with $\bar{R}_{\mathrm{Opt}}$

Fig. 2 shows $\bar{R}_{\text {Opt }}$ versus the overhearing channel load for the low user density scenario in Fig. 1. The optimal choice of $\lambda$ and $\theta$, as well as the optimal RZF regularizer, is found at each point by maximizing the asymptotic limit in (49). The 


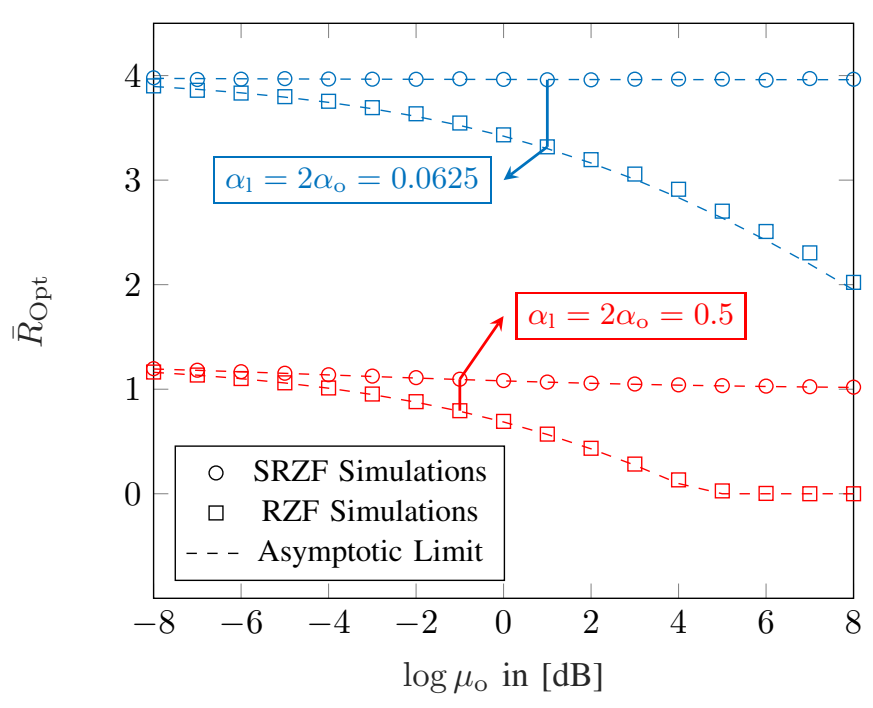

Fig. 3: Optimized average ergodic secrecy rate vs. the receive SNR at the eavesdroppers. The simulations are given for $M=128$ and $\log \mu_{1}=0 \mathrm{~dB}$.

simulation points are then calculated by simulating the system with optimally tuned parameters for $M=128$.

Considering Fig. 2, two findings are demonstrated: 1) For non-zero overhearing channel loads, the SRZF scheme constantly outperforms RZF. This finding is intuitive, since for $\alpha_{\mathrm{o}} \neq 0$, the so-called secrecy-for-free property, reported in [6], does not hold anymore, and hence, further suppression of leakage by SRZF enhances the performance. 2) The gap between the secrecy rates achieved by SRZF and RZF increase as $\alpha_{\mathrm{o}}$ grows. This observation comes from the fact that as in networks with high density of eavesdroppers, beamforming based on the legitimate channel information results in high information leakage, and hence modification of the beamformers based on the eavesdropper channel improves the secrecy performance considerably.

\section{B. Robustness of SRZF Precoding}

To study the further gains proposed by the SRZF scheme, we plot the optimized average ergodic secrecy rate against the receive SNR at the eavesdroppers, i.e. $\mu_{\mathrm{o}}$, for the two scenarios, considered in Fig. 1, when $\alpha_{\mathrm{o}}=\alpha_{\mathrm{l}} / 2$. In this figure, $\log \mu_{\mathrm{l}}=0 \mathrm{~dB}$, and $\log \mu_{\mathrm{o}}$ is swept between -8 and 8 $\mathrm{dB}$. The figure depicts that the average secrecy rate achieved by the RZF scheme drops significantly, as the receive SNR at the eavesdroppers increases. The SRZF scheme however is very robust. This phenomena demonstrates the efficiency of beamforming modification proposed by SRZF and indicates its robustness against the quality of eavesdroppers' channels.

\section{CONCLUSIONS}

A novel linear precoding scheme has been proposed for downlink transmission in MIMOME channels. A closed-form expression for the asymptotic achievable secrecy rate per user has been derived for this precoder. The large-system results depict tight consistency with simulations, and hence can be employed to tune the precoder. Numerical investigations have demonstrated high performance enhancements achieved by the proposed scheme. Specifically, the precoder has shown to be highly robust against the change in the channel quality and outperform significantly the well-known RZF scheme when the eavesdroppers experience better channel quality compared to the legitimate receivers.

\section{REFERENCES}

[1] A. Khisti and G. W. Wornell, "Secure transmission with multiple antennas-Part II: The MIMOME wiretap channel," IEEE Transactions on Information Theory, vol. 56, no. 11, pp. 5515-5532, 2010.

[2] G. Geraci, M. Egan, J. Yuan, A. Razi, and I. B. Collings, "Secrecy sumrates for multi-user MIMO regularized channel inversion precoding," IEEE Transactions on Communications, vol. 60, no. 11, pp. 3472-3482, 2012.

[3] G. Geraci, A. Y. Al-nahari, J. Yuan, and I. B. Collings, "Linear precoding for broadcast channels with confidential messages under transmitside channel correlation," arXiv preprint arXiv:1304.6157, 2013.

[4] G. Geraci, R. Couillet, J. Yuan, M. Debbah, and I. B. Collings, "Large system analysis of linear precoding in MISO broadcast channels with confidential messages," IEEE Journal on Selected Areas in Communications, vol. 31, no. 9, pp. 1660-1671, 2013.

[5] D. Kapetanovic, G. Zheng, and F. Rusek, "Physical layer security for massive MIMO: An overview on passive eavesdropping and active attacks," IEEE Communications Magazine, vol. 53, no. 6, pp. 21-27, 2015.

[6] A. Bereyhi, S. Asaad, R. R. Müller, R. F. Schaefer, and A. M. Rabiei, "On robustness of massive MIMO systems against passive eavesdropping under antenna selection," IEEE Global Communications Conference (GLOBECOM), 2018.

[7] J. Zhu, R. Schober, and V. K. Bhargava, "Secure transmission in multicell massive MIMO systems," IEEE Transactions on Wireless Communications, vol. 13, no. 9, pp. 4766-4781, 2014.

[8] A. Bereyhi, M. A. Sedaghat, R. R. Müller, and G. Fischer, "GLSE precoders for massive MIMO systems: Analysis and applications," IEEE Transactions on Wireless Communications, vol. 18, no. 9, pp. 44504465, 2019.

[9] A. Bereyhi, M. A. Sedaghat, and R. R. Müller, "Asymptotics of nonlinear LSE precoders with applications to transmit antenna selection," in IEEE International Symposium on Information Theory (ISIT), 2017, pp. $81-85$.

[10] A. Bereyhi, M. A. Sedaghat, S. Asaad, and R. R. Müller, "Nonlinear precoders for massive MIMO systems with general constraints," International ITG Workshop on Smart Antennas (WSA), VDE, 2017.

[11] C. B. Peel, B. M. Hochwald, and A. L. Swindlehurst, "A vector-perturbation technique for near-capacity multiantenna multiuser communication-Part I: channel inversion and regularization," IEEE Transactions on Communications, vol. 53, no. 1, pp. 195-202, 2005.

[12] V. K. Nguyen and J. S. Evans, "Multiuser transmit beamforming via regularized channel inversion: A large system analysis," in IEEE Global Communications Conference (GLOBECOM), 2008.

[13] E. Ekrem and S. Ulukus, "The secrecy capacity region of the Gaussian MIMO multi-receiver wiretap channel," IEEE Transactions on Information Theory, vol. 57, no. 4, pp. 2083-2114, 2011.

[14] A. M. Tulino and S. Verdú, "Random matrix theory and wireless communications," Foundations and Trends in Communications and Information Theory, vol. 1, no. 1, pp. 1-182, 2004.

[15] R. R. Müller, G. Alfano, B. M. Zaidel, and R. de Miguel, "Applications of large random matrices in communications engineering," arXiv preprint arXiv:1310.5479, 2013.

[16] J. W. Silverstein and Z. Bai, "On the empirical distribution of eigenvalues of a class of large dimensional random matrices," Journal of Multivariate analysis, vol. 54, no. 2, pp. 175-192, 1995. 